\title{
Generalized semiconfined harmonic oscillator model with a position-dependent effective mass
}

\author{
C. Quesne* \\ Physique Nucléaire Théorique et Physique Mathématique, Université Libre de Bruxelles, \\ Campus de la Plaine CP229, Boulevard du Triomphe, B-1050 Brussels, Belgium
}

\begin{abstract}
By using a point canonical transformation starting from the constant-mass Schrödinger equation for the isotonic potential, it is shown that a semiconfined harmonic oscillator model with a position-dependent mass in the BenDanielDuke setting and the same spectrum as the standard harmonic oscillator can be easily constructed and extended to a semiconfined shifted harmonic oscillator, which could result from the presence of a uniform gravitational field. A further generalization is proposed by considering a $m$-dependent positiondependent mass for $0<m<2$ and deriving the associated semiconfined potential. This results in a family of position-dependent mass and potential pairs, to which the original pair belongs as it corresponds to $m=1$. Finally, the potential that would result from a general von Roos kinetic energy operator is presented and the examples of the Zhu-Kroemer and MustafaMazharimousavi settings are briefly discussed.
\end{abstract}

Keywords: Schrödinger equation; position-dependent mass; harmonic oscillator; point canonical transformation

PACS Nos.: 03.65.Fd, 03.65.Ge

${ }^{*}$ e-mail: Christiane.Quesne@ulb.be 


\section{Introduction}

There is much interest in the Schrödinger equation wherein the constant mass is replaced by a position-dependent mass (PDM), because the latter has many applications in problems occurring in several fields of physics [1, 2, 3, 4, 5, 6, 7, 8, 9, 10, 11, 12. As it has been shown [13] that the PDM Schrödinger equation is equivalent to two other unconventional Schrödinger equations, namely the Schrödinger equation resulting from the use of deformed commutation relations [14, 15, 16, as well as that in curved space [17, 18, 19], this has reinforced the interest in its study.

As a consequence, much attention has been devoted to finding exact solutions of PDM Schrödinger equations because they may provide a conceptual understanding of some physical phenomena, as well as a testing ground for some approximation schemes. The generation of PDM and potential pairs leading to such exact solutions has been achieved by various methods (see, e.g., [20] and references quoted therein). One of the most powerful techniques for such a purpose consists in applying a point canonical transformation (PCT) to an exactly solvable constant-mass Schrödinger equation [21, 22]. Recently, such an approach has proved its efficiency again by providing a straightforward generalization [23] of a harmonic oscillator model wherein both the mass and the angular frequency are dependent on the position [24].

The purpose of the present paper is to re-examine a new model of semiconfined harmonic oscillator with a mass that varies with position, which has the striking property of having the same spectrum as the standard harmonic oscillator model [25]. By using the PCT method, we plan to prove that one can find a family of PDM and semiconfined potential pairs corresponding to such a spectrum and to which the original PDM and semiconfined harmonic oscillator pair belongs.

The paper is organized as follows. In Sec. 2, the model of [25] is reviewed and shown to be derivable by applying the PCT technique to the constant-mass isotonic oscillator model [26, 27]. In Sec. 3, an extension of the model is proposed by starting from a more general PDM and determining the associated semiconfined potential. Finally, Sec. 4 contains some comments. 


\section{Semiconfined harmonic oscillator model and its derivation by the PCT technique}

Jafarov and Van der Jeugt recently determined the exact solution of a PDM semiconfined harmonic oscillator model, characterized by the Schrödinger equation [25]

$$
\left(-\frac{d}{d x} \frac{1}{M(x)} \frac{d}{d x}+V_{\mathrm{eff}}(x)\right) \psi_{n}(x)=E_{n} \psi_{n}(x)
$$

where the kinetic energy operator has the BenDaniel-Duke form [28] and the potential has the harmonic oscillator form

$$
V_{\mathrm{eff}}(x)=\frac{1}{4} M(x) \omega^{2} x^{2}
$$

except that the mass

$$
M(x)= \begin{cases}\left(1+\frac{x}{a}\right)^{-1} & \text { if }-a<x<+\infty \\ +\infty & \text { if } x \leq-a\end{cases}
$$

with $a>0$, depends on the position in such a way that $V_{\text {eff }}(-a)=+\infty$ and $\lim _{x \rightarrow+\infty} V_{\text {eff }}(x)=+\infty 1$

By directly solving the differential equation (1), they found that the spectrum of this semiconfined model is that of the standard harmonic oscillator,

$$
E_{n}=\omega\left(n+\frac{1}{2}\right), \quad n=0,1,2, \ldots,
$$

with corresponding wavefunctions

$$
\psi_{n}(x)=C_{n}\left(1+\frac{x}{a}\right)^{\frac{1}{2} \omega a^{2}} e^{-\frac{1}{2} \omega a(x+a)} L_{n}^{\left(\omega a^{2}\right)}\left(\omega a^{2}\left(1+\frac{x}{a}\right)\right), \quad-a<x<+\infty,
$$

expressed in terms of Laguerre polynomials $L_{n}^{(\alpha)}(z)$ and vanishing at $x=-a$ and $x \rightarrow+\infty$, as it should be. Here $C_{n}$ is a normalization coefficient given by

$$
C_{n}=\left(\omega a^{2}\right)^{\frac{1}{2}\left(\omega a^{2}+1\right)} \sqrt{\frac{n !}{a \Gamma\left(\omega a^{2}+n+1\right)}} .
$$

\footnotetext{
${ }^{1}$ Note that we have adopted here units wherein $\hbar=2 m_{0}$ in the original paper.
} 
These results may be alternatively derived by applying a PCT to the constantmass Schrödinger equation for the isotonic oscillator [26, 27]

$$
\left(-\frac{d^{2}}{d u^{2}}+U(u)\right) \phi_{n}(u)=\epsilon_{n} \phi_{n}(u)
$$

where

$$
\begin{gathered}
U(u)=\frac{1}{4} \bar{\omega}^{2} u^{2}+\frac{g}{u^{2}}, \quad g>0, \quad 0<u<+\infty, \\
\epsilon_{n}=\bar{\omega}(2 n+\alpha+1), \quad \alpha=\frac{1}{2} \sqrt{1+4 g}
\end{gathered}
$$

and

$$
\phi_{n}(u) \propto u^{\alpha+\frac{1}{2}} e^{-\frac{1}{4} \bar{\omega} u^{2}} L_{n}^{(\alpha)}\left(\frac{1}{2} \bar{\omega} u^{2}\right) .
$$

A PCT transforming an equation such as (77) into a PDM equation of type (1) [21, 22] consists in making a change of variable

$$
u(x)=\bar{a} v(x)+\bar{b}, \quad v(x)=\int^{x} \sqrt{M\left(x^{\prime}\right)} d x^{\prime},
$$

and a change of function

$$
\phi_{n}(u(x)) \propto[M(x)]^{-1 / 4} \psi_{n}(x) .
$$

The potential $V_{\text {eff }}(x)$ and the energy eigenvalues $E_{n}$ of the PDM Schrödinger equation are then given in terms of the potential and the energy eigenvalues of the constant-mass one by

$$
V_{\text {eff }}(x)=\bar{a}^{2} U(u(x))+\frac{M^{\prime \prime}}{4 M^{2}}-\frac{7 M^{\prime 2}}{16 M^{3}}+\bar{c},
$$

and

$$
E_{n}=\bar{a}^{2} \epsilon_{n}+\bar{c}
$$

where a prime denotes derivation with respect to $x$ and $\bar{a}, \bar{b}, \bar{c}$ are three real constants.

In the present case, from (3) and (11), we directly obtain

$$
v(x)=2 a \sqrt{1+\frac{x}{a}} .
$$


and

$$
\frac{M^{\prime \prime}}{4 M^{2}}-\frac{7 M^{\prime 2}}{16 M^{3}}=\frac{1}{16 a^{2}}\left(1+\frac{x}{a}\right)^{-1}
$$

for $-a<x<+\infty$. With the choice $\bar{a}=\sqrt{\frac{\omega}{2 \bar{\omega}}}, \bar{b}=0$, we get for the change of variable (11)

$$
u(x)=a \sqrt{\frac{2 \omega}{\bar{\omega}}} \sqrt{1+\frac{x}{a}}
$$

and the change of function (12), together with (10), leads to

$$
\psi_{n}(x)=C_{n}\left(1+\frac{x}{a}\right)^{\alpha / 2} e^{-\frac{1}{2} \omega a(x+a)} L_{n}^{(\alpha)}\left(\omega a^{2}\left(1+\frac{x}{a}\right)\right),
$$

where $C_{n}$ turns out to be

$$
C_{n}=\left(\omega a^{2}\right)^{\frac{1}{2}(\alpha+1)} \sqrt{\frac{n !}{a \Gamma(\alpha+n+1)}} .
$$

Furthermore, on assuming $\bar{c}=-\frac{\omega}{2} \alpha$, the transformed potential (13) becomes

$$
V_{\text {eff }}(x)=\frac{a \omega^{2}}{4(x+a)}\left(x+a-\frac{\alpha}{a \omega}\right)^{2},
$$

with corresponding eigenvalues $E_{n}$ given by (4).

If we compare these results with those of [25], we notice that we have obtained the same energy spectrum (4), but with generalized potential and wavefunctions, since the latter depend on an extra parameter $\alpha$ absent in [25]. By taking $\alpha=a^{2} \omega$, the original results are retrieved, but for other values of $\alpha$, the potential (20) describes a semiconfined shifted harmonic oscillator. Note that such a potential might be interpreted as a semiconfined harmonic oscillator in a uniform gravitational field as was done for a shifted harmonic oscillator with another type of PDM [29, 30].

\section{Family of generalized semiconfined oscillator models}

A further generalization of the model of [25] can be obtained by changing the PDM (3) into a PDM depending on some parameter $m$ taking values in the interval $0<$ $m<2$,

$$
M(x)= \begin{cases}\left(1+\frac{x}{a}\right)^{-m} & \text { if }-a<x<+\infty \\ +\infty & \text { if } x \leq-a\end{cases}
$$


and determining the associated potential $V_{\text {eff }}(x)$ with the assumption that the starting constant-mass Schrödinger equation remains as given in (7) and (8). The results of Sec. 2 will then correspond to the $m=1$ special case.

Equations (15) and (16) are now replaced by

$$
v(x)=\frac{2 a}{2-m}\left(1+\frac{x}{a}\right)^{1-\frac{m}{2}}
$$

and

$$
\frac{M^{\prime \prime}}{4 M^{2}}-\frac{7 M^{\prime 2}}{16 M^{3}}=-\frac{1}{16 a^{2}} m(3 m-4)\left(1+\frac{x}{a}\right)^{m-2}
$$

respectively. On keeping the same values for $\bar{a}, \bar{b}$, and $\bar{c}$ as in Sec. 2, we get a new change of variable

$$
u(x)=\frac{a}{2-m} \sqrt{\frac{2 \omega}{\bar{\omega}}}\left(1+\frac{x}{a}\right)^{1-\frac{m}{2}}
$$

but the resulting energy eigenvalues remain given by (44). From (13), however, the resulting potential turns out to be $m$-dependent and given by

$$
\begin{aligned}
V_{\mathrm{eff}}(x)= & \frac{a^{m} \omega^{2}}{4(2-m)^{2}}(x+a)^{2-m}+\frac{[(m-2) \alpha-(m-1)][(m-2) \alpha+m-1]}{4 a^{m}(x+a)^{2-m}} \\
& -\frac{1}{2} \omega \alpha .
\end{aligned}
$$

This is also the case for the wavefunctions, which become

$$
\begin{aligned}
\psi_{n}(x)= & C_{n}\left(1+\frac{x}{a}\right)^{-\frac{m}{2}(\alpha+1)+\alpha+\frac{1}{2}} e^{-\frac{1}{2(2-m)^{2}} \omega a^{2}\left(1+\frac{x}{a}\right)^{2-m}} \\
& \times L_{n}^{(\alpha)}\left(\frac{\omega a^{2}}{(2-m)^{2}}\left(1+\frac{x}{a}\right)^{2-m}\right)
\end{aligned}
$$

where

$$
C_{n}=\left(\frac{\omega a^{2}}{(2-m)^{2}}\right)^{\frac{1}{2}(\alpha+1)} \sqrt{\frac{(2-m) n !}{a \Gamma(\alpha+n+1)}} .
$$

The new $m$-dependent potential (25) will be a semiconfined potential provided it goes to $+\infty$ for $x \rightarrow+\infty$ and $x \rightarrow-a$. The former condition is automatically satisfied, but the latter imposes that

$$
\alpha>\frac{m-1}{2-m}
$$




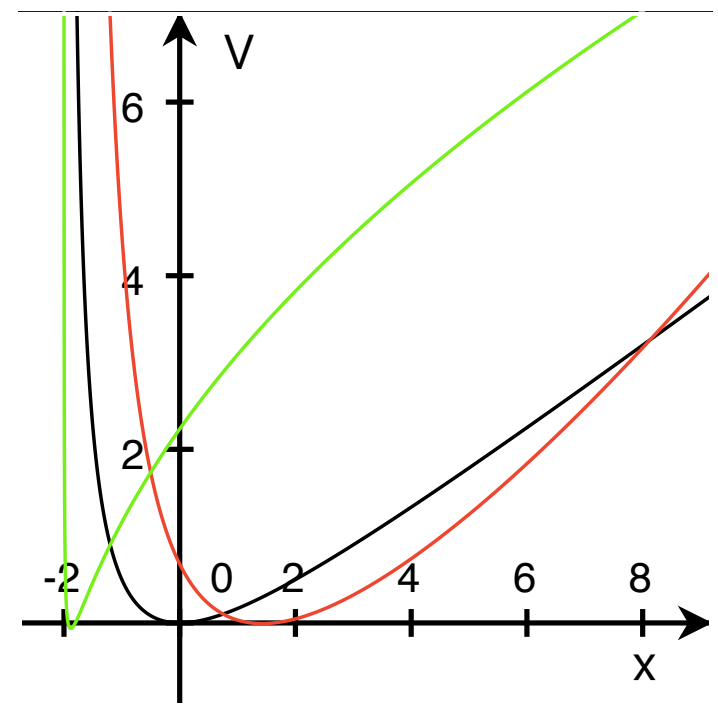

Figure 1: Plot of the semiconfined potential (25) in terms of $x$ for $m=1$ (black line), $m=\frac{1}{2}$ (red line), and $m=\frac{3}{2}$ (green line). The parameter values are $\omega=1$, $a=2$, and $\alpha=4$.

which implies a restriction for $m$ values such that $\frac{m-1}{2-m}>\frac{1}{2}$, i.e., for those in the interval $\frac{4}{3}<m<2$. In such a case, the wavefunctions (26) vanish for $x \rightarrow+\infty$ and $x \rightarrow-a$, as it should be. The minimum of the potential occurs for

$$
x_{\min }=-a+\left\{\frac{(2-m)^{2}}{a^{m} \omega} \sqrt{\alpha^{2}-\left(\frac{m-1}{2-m}\right)^{2}}\right\}^{1 /(2-m)}
$$

and is given by

$$
\left(V_{\mathrm{eff}}\right)_{\min }=\frac{1}{2} \omega\left\{\sqrt{\alpha^{2}-\left(\frac{m-1}{2-m}\right)^{2}}-\alpha\right\} .
$$

It is therefore slightly negative, except for $m=1$ for which it vanishes.

In Fig. 1, we show the dependence of the semiconfined potential (25) on $m$. The black line corresponds to the original semiconfined harmonic oscillator (2).

\section{Comments}

In the present paper, we have first shown that the PCT method applied to the constant-mass Schrödinger equation for the isotonic oscillator allows us to easily 
retrieve the results of [25] and to extend them in order to describe a semiconfined shifted harmonic oscillator, which might be interpreted as a semiconfined harmonic oscillator in a uniform gravitational field.

In a second step, we have obtained a further generalization by considering a $m$-dependent PDM for $0<m<2$ and by deriving the corresponding semiconfined potential with the same spectrum as the standard harmonic oscillator. We have therefore constructed a family of PDM and potential pairs, to which the original pair belongs as it corresponds to $m=1$.

In [25], the BenDaniel-Duke ordering [28] was chosen for the momentum and mass operators. One finds, however, in the literature, several other orderings, which are special cases of the von Roos general two-parameter form of the kinetic energy operator [31], for which the Schrödinger operator writes

$$
\begin{aligned}
& \left\{-\frac{1}{2}\left[M(x)^{\xi} \frac{d}{d x} M(x)^{\eta} \frac{d}{d x} M(x)^{\zeta}+M(x)^{\zeta} \frac{d}{d x} M(x)^{\eta} \frac{d}{d x} M(x)^{\xi}\right]+V_{\mathrm{vR}}(x)\right\} \psi_{n}(x) \\
& \quad=E_{n} \psi_{n}(x)
\end{aligned}
$$

where $\xi, \eta, \zeta$ are some real parameters restricted by the condition $\xi+\eta+\zeta=-1$. In particular, the BenDaniel-Duke ordering corresponds to $\xi=\zeta=0, \eta=-1$ and the relation between the potentials in (1) and (31) is given by

$$
V_{\mathrm{vR}}(x)=V_{\mathrm{eff}}(x)-\frac{1}{2}(1+\eta) \frac{M^{\prime \prime}}{M^{2}}+[\xi(\xi+\eta+1)+\eta+1] \frac{M^{\prime 2}}{M^{3}} .
$$

For the mass chosen in (21), the latter becomes

$$
\begin{aligned}
& V_{\mathrm{vR}}(x) \\
& =V_{\mathrm{eff}}(x)+\left\{-\frac{1}{2}(1+\eta) m(m+1)+[\xi(\xi+\eta+1)+\eta+1] m^{2}\right\} \frac{(x+a)^{m-2}}{a^{m}}
\end{aligned}
$$

It is worth noting, in particular, the Zhu-Kroemer [32] and MustafaMazharimousavi [33] orderings, which pass the de Souza Dutra and Almeida test [34] as good orderings. The former corresponds to $\xi=\zeta=-\frac{1}{2}, \eta=0$, and leads to replacing (33) by

$$
V_{\mathrm{ZK}}(x)=\frac{a^{m} \omega^{2}}{4(2-m)^{2}}(x+a)^{2-m}+\frac{[(m-2) \alpha-1][(m-2) \alpha+1]}{4 a^{m}(x+a)^{2-m}}-\frac{1}{2} \omega \alpha,
$$


while the latter is associated with $\xi=\zeta=-\frac{1}{4}, \eta=-\frac{1}{2}$, and gives rise to

$$
V_{\mathrm{MM}}(x)=\frac{a^{m} \omega^{2}}{4(2-m)^{2}}(x+a)^{2-m}+\frac{(m-2)^{2}\left(\alpha^{2}-\frac{1}{4}\right)}{4 a^{m}(x+a)^{2-m}}-\frac{1}{2} \omega \alpha .
$$

These potentials have a behaviour very similar to that of $V_{\text {eff }}(x)$, since they are semiconfined for $\alpha$ restricted to $\alpha>1 /(2-m)$ or for any value of $\alpha(>1 / 2$ by definition (9) ), respectively. The place of the minimum and its value are given by (29) and (30) provided $\sqrt{\alpha^{2}-[(m-1) /(2-m)]^{2}}$ is replaced by $\sqrt{\alpha^{2}-1 /(2-m)^{2}}$ or $\sqrt{\alpha^{2}-1 / 4}$.

\section{Acknowledgements}

This work was supported by the Fonds de la Recherche Scientifique-FNRS under Grant No. 4.45.10.08. 


\section{References}

[1] G. Bastard, Wave Mechanics Applied to Semiconductor Heterostructures (Editions de Physique, Les Ulis, 1988)

[2] C. Weisbuch, B. Vinter, Quantum Semiconductor Heterostructures (Academic, New York, 1997)

[3] L. Serra, E. Lipparini, Spin response of unpolarized quantum dots. Europhys. Lett. 40, 667 (1997)

[4] P. Harrison, A. Valavanis, Quantum Wells, Wires and Dots: Theoretical and Computational Physics of Semiconductor Nanostructures (Wiley, Chichester, 2016)

[5] M. Barranco, M. Pi, S.M. Gatica, E.S. Hernández, J. Navarro, Structure and energetics of mixed ${ }^{4} \mathrm{He}-{ }^{3} \mathrm{He}$ drops. Phys. Rev. B 56, 8997 (1997)

[6] M.R. Geller, W. Kohn, Quantum mechanics of electrons in crystals with graded composition. Phys. Rev. Lett. 70, 3103 (1993)

[7] F. Arias de Saavedra, J. Boronat, A. Polls, A. Fabrocini, Effective mass of one ${ }^{4}$ He atom in liquid ${ }^{3}$ He. Phys. Rev. B 50, 4248(R) (1994)

[8] A. Puente, L. Serra, M. Casas, Dipole excitation of Na clusters with a non-local energy density functional. Z. Phys. D 31, 283 (1994)

[9] P. Ring, P. Schuck, The Nuclear Many Body Problem (Springer, New York, 1980)

[10] D. Bonatsos, P.E. Georgoudis, D. Lenis, N. Minkov, C. Quesne, Bohr Hamiltonian with a deformation-dependent mass term for the Davidson potential. Phys. Rev. C 83, 044321 (2011)

[11] W. Willatzen, B. Lassen, The BenDaniel-Duke model in general nanowire structures. J. Phys. Condens. Matter 19, 136217 (2007) 
[12] N. Chamel, Effective mass of free neutrons in neutron star crust. Nucl. Phys. A 773, 263 (2006)

[13] C. Quesne, V.M. Tkachuk, Deformed algebras, position-dependent effective mass and curved spaces: An exactly solvable Coulomb problem. J. Phys. A: Math. Gen. 37, 4267 (2004)

[14] A. Kempf, Uncertainty relation in quantum mechanics with quantum group symmetry. J. Math. Phys. 35, 4483 (1994)

[15] H. Hinrichsen, A. Kempf, Maximal localization in the presence of minimal uncertainties in positions and in momenta. J. Math. Phys. 37, 2121 (1996)

[16] E. Witten, Reflections on the fate of spacetime. Phys. Today 49, 24 (1996)

[17] E. Schrödinger, A method of determining quantum-mechanical eigenvalues and eigenfunctions. Proc. R. Ir. Acad. A46, 9 (1940)

[18] E.G. Kalnins, W. Miller Jr., G.S. Pogosyan, Superintegrability and associated polynomial solutions: Euclidean space and the sphere in two dimensions. J. Math. Phys. 37, 6439 (1996)

[19] E.G. Kalnins, W. Miller Jr., G.S. Pogosyan, Superintegrability on the twodimensional hyperboloid. J. Math. Phys. 38, 5416 (1997)

[20] C. Quesne, First-order intertwining operators and position-dependent mass Schrödinger equations in $d$ dimensions. Ann. Phys. (NY) 321, 1221 (2006)

[21] B. Bagchi, P. Gorain, C. Quesne, R. Roychoudhury, A general scheme for the effective-mass Schrödinger equation and the generation of the associated potentials. Mod. Phys. Lett. A 19, 2765 (2004)

[22] C. Quesne, Point canonical transformation versus deformed shape invariance for position-dependent mass Schrödinger equations. SIGMA 5, 046 (2009) 
[23] C. Quesne, Comment on 'Exact solution of the position-dependent effective mass and angular frequency Schrödinger equation: harmonic oscillator model with quantized confinement parameter'. J. Phys. A: Math. Theor. 54, 368001 $(2021)$

[24] E.I. Jafarov, S.M. Nagiyev, R. Oste, J. Van der Jeugt, Exact solution of the position-dependent effective mass and angular frequency Schrödinger equation: harmonic oscillator model with quantized confinement parameter. J. Phys. A: Math. Theor. 53, 485301 (2020)

[25] E.I. Jafarov, J. Van der Jeugt, Exact solution of the semiconfined harmonic oscillator model with a position-dependent effective mass. Eur. Phys. J. Plus 136, $758(2021)$

[26] Y. Weissman, J. Jortner, The isotonic oscillator. Phys. Lett. A 70, 177 (1979)

[27] D. Zhu, A new potential with the spectrum of an isotonic oscillator. J. Phys. A: Math. Gen. 20, 4331 (1987)

[28] D.J. BenDaniel, C.B. Duke, Space-charge effects on electron tunneling. Phys. Rev. 152, 683 (1966)

[29] E.I. Jafarov, S.M. Nagiyev, A.M. Jafarova, Quantum-mechanical explicit solution for the confined harmonic oscillator model with the von Roos kinetic energy operator. Rep. Math. Phys. 86, 25 (2020)

[30] E.I. Jafarov, S.M. Nagiyev, Angular part of the Schrödinger equation for the Hautot potential as a harmonic oscillator with a coordinate-dependent mass in a uniform gravitational field. Theor. Math. Phys. 207, 447 (2021)

[31] O. von Roos, Position-dependent effective masses in semiconductor theory. Phys. Rev. B 27, 7547 (1983) 
[32] Q.-G. Zhu, H. Kroemer, Interface connection rules for effective-mass wave functions at an abrupt heterojunction between two different semiconductors. Phys. Rev. B 27, 3519 (1983)

[33] O. Mustafa, S.H. Mazharimousavi, Ordering ambiguity revisited via position dependent mass pseudo-momentum operators. Int. J. Theor. Phys. 46, 1786 (2007)

[34] A. de Souza Dutra, C.A.S. Almeida, Exact solvability of potentials with spatially dependent effective masses. Phys. Lett. A 275, 25 (2000) 